\title{
Perfil de jugador virtual asociado a la motivación personal para jugar en CityVille
}

\section{Virtual player profile associated with personal motivation to play in City Ville}

\author{
$\mathrm{M}^{\mathrm{a}}$ Esther del Moral-Pérez \\ Universidad de Oviedo. Oviedo, España \\ emoral@uniovi.es \\ Alba Patricia Guzmán-Duque \\ Unidades Tecnológicas de Santander. Santander, Colombia \\ aguzman@correo.uts.edu.co
}

\begin{abstract}
Resumen
Esta investigación describe la comunidad Fans-CityVille $(\mathrm{N}=110)$ en función de las variables sexo, tiempo dedicado al juego, nivel alcanzado y dinero invertido. Asimismo, identifica los tres perfiles de jugador virtual asociados con las motivaciones esgrimidas para permanecer en el juego, los objetivos priorizados en el mismo y las razones alegadas para interactuar con otros jugadores: a) utilitarista-adictivo, b) aprendizaje lúdico-colaborativo, y c) prosocial. Entre los resultados se observa que los usuarios juegan para divertirse interaccionando online y colaborando con otros para hacer crecer sus ciudades en CityVille, son felices jugando y compartiendo afición. Aproximadamente la mitad juega entre 3-6 horas diarias y, aunque se podía jugar sin gastar dinero, casi la mitad invertía una media de 8 mensuales para avanzar más rápido. Los hombres se muestran más utilitaristas, buscan alcanzar el máximo nivel y solo interactúan con otros jugadores para obtener beneficios y ganar; a diferencia de las mujeres que valoran el aprendizaje derivado de este entretenimiento colaborativo y prosocial, ponderan más el intercambio de estrategias, la colaboración mutua y la oportunidad que les brinda el juego para ampliar su red de contactos y amistades.
\end{abstract}

Palabras clave

CityVille, juegos en red social, motivación personal, perfil de jugador.

\begin{abstract}
This research describes the Community Fans-Cityville $(\mathrm{N}=110)$ depending on the variables sex, time dedicated to the game, level reached and money invested. It also identifies the three player profiles associated with the given motivations to remain in the game, the prioritized objectives in the game and the alleged reasons to interact with other players: a) utilitarian-addictive, b) recreational-collaborative learning, and c) prosocial. Among the results it is observed that the players have fun interacting online and collaborating with others to grow their cities, they are happy playing and sharing hobby. Almost half play between 3-6 hours a day in CityVille and, although you could play without spending money, almost half spend an average of $\$ 8$ monthly to advance faster. Men are more utilitarian, seek to reach the highest level and only interact with others to gain and win; Unlike women who prioritize collaborative and prosocial entertainment, they value the exchange of strategies, mutual collaboration and the opportunity that the game offers to broaden their network of contacts and friendships.
\end{abstract}

Key words

CityVille, Social Network Games, Personal Motivation, Player profile 


\section{Introducción}

Dentro de las redes sociales surgieron plataformas específicas diseñadas para jugar online, las cuales favorecieron la conversión de los usuarios en jugadores virtuales, al invitarles a sumarse al fenómeno del entretenimiento colaborativo animados por sus contactos, lo que ha dado lugar a una nueva cultura de juego online (Guegan, Moliner \& Buisine, 2015). Estas prácticas lúdicas - insertas en atractivos escenarios- se han ido consolidando en internet al permitir el juego multijugador, aprovechando las conexiones de los propios usuarios a través de Facebook, Google+, etc. Su éxito radica en la oportunidad que ofrecen para congregar a los numerosos habitantes de la red (Correa, 2016), fomentando su interconexión e impulsando su participación en torneos y juegos diversos. Lo que les está confiriendo una dimensión planetaria, que algunas empresas han sabido explotar como negocio (De Andrade, 2012; Feinleib, 2011).

Así, se observa que se han popularizado juegos que promueven prácticas lúdicas virtuales de distinta índole, invitando a los jugadores a comunicarse con otros para alcanzar sus fines (Burroughs, 2014). Algunos exigen la interacción y el apoyo mutuo para promocionar o tener éxito, rompiendo con el cuestionado individualismo y la feroz competitividad que les estigmatizaba. Tal fue el caso del éxito de los juegos en red con un eminente carácter prosocial, ligados a la construcción de granjas (FarmVille), castillos (CastleVille), ciudades (CityVille), etc., cuyo valor añadido radicaba en ofrecer actividades que fomentaban la adquisición de conocimientos específicos, la colaboración y la transferencia de estrategias entre los jugadores virtuales, tanto para ampliar sus dominios como adquirir un mayor rango, etc. En ellos se primaba el aprendizaje a partir de la experiencia de juego compartido, al establecer pautas de interacción ligadas a la cooperación y al apoyo entre los usuarios para superar retos y promocionar de nivel (Camilleri, Busuttil \& Montebello, 2011).

Sin duda, este tipo de juegos en red logran implicar a los usuarios tanto a nivel cognitivo como motivacional, emocional y social (Granic, Lobel \& Engels, 2014). Poseen un extraordinario poder para involucrarlos (Berle, Starcevic, Porter \& Fenech, 2015; Kelly, 2010), activando su inmersión a partir de la interacción real con otros (Chiang, Lin, Cheng \& Liu, 2011), e incrementando su nivel de satisfacción al proponerles colaborar en tareas voluntarias de forma puntual y alentar conductas prosociales (You, Kim \& Lee, 2017). Evidentemente, la posibilidad de establecer lazos reales con otros usuarios de la red, a propósito de tareas compartidas en un juego online, supone una gran motivación al asumir roles complementarios, no excluyentes, adscritos a las técnicas de negociación ganar-ganar (Guenaga, Eguíluz, Jerez \& Torientes, 2015).

En estos contextos lúdicos virtuales, donde se prioriza el intercambio y la interacción, se generan interesantes relaciones, afiliaciones e incluso, comunidades espontáneas en torno a usuarios con cierta reputación social adquirida en el juego. Por otro lado, desde una perspectiva psicológica, se observa cómo el comportamiento humano se ve condicionado por las dinámicas y mecánicas de cada juego, así como por el tipo de interacciones que se priorizan, y ello se percibe en las motivaciones, objetivos y prioridades que establecen los usuarios para jugar, interactuar y comunicarse (Choi, Choi \& Song, 2012; Guegan, Moliner \& Buisine, 2015).

Perfil de jugador virtual asociado a la motivación personal para jugar en CityVille. M. Esther del Moral Pérez y Alba Patricia Guzmán Duque. 
Así pues, esta investigación indaga en las razones que impulsan a los sujetos a estar en juegos orientados a la construcción virtual online y a interactuar con otros usuarios, de modo similar a como hicieron Cole y Griffiths (2007), pero en este caso para identificar los perfiles más destacados de los jugadores (Andreassen, Billieux, Griffiths, Kuss, Demetrovics, Mazzoni \& Pallesen, 2016). En concreto, el juego seleccionado fue CityVille, por considerarlo -en su momento- capaz de propiciar aprendizajes de carácter socio-cognitivo y desarrollar distintas habilidades a partir de la colaboración y del intercambio de estrategias para la construcción de ciudades virtuales (Coelho, Espinosa \& Medina, 2013; Del Moral \& Guzmán, 2014). Para ello, inicialmente se describen las mecánicas y dinámicas del juego claves para fidelizar a los jugadores, y más tarde, se analiza la relación de las variables propias de su condición de jugador (género, tiempo, gasto invertido en el juego, etc.) con las motivaciones que les llevan a permanecer en el juego, los objetivos fijados para avanzar y las prioridades establecidas para comunicarse e interaccionar con otros, etc., lo que permite determinar tres tipos de perfiles de jugadores.

\section{CityVille: narrativa centrada en la construcción de ciudades}

CityVille (CV) -lanzado en 2010 en Facebook, en español, inglés, francés, alemán y japonés- llegó a contar con más de 17 millones de usuarios (Zynga, 2013) -cerrado en abril 2015-. El juego se apoyaba en la estrategia de simulación para crear ciudades virtuales, suscitando aprendizajes muy diversos (Nummenmaa, Alha \& Kultima, 2011). Proponía la construcción de casas, negocios, edificios comunitarios, gubernamentales, etc., donde el incremento de habitantes era un factor clave para promocionar de nivel. Favorecía las relaciones de colaboración entre jugadores y el reconocimiento de la experiencia ajena, al gratificar las visitas a las ciudades de los vecinos (Huang, Ali \& Liao, 2017). Alentaba a los usuarios para que invitasen a sus conocidos a jugar y promovía las relaciones con nuevos jugadores. Al activar los contactos se aumentaba la recaudación, la energía y, lógicamente, el crecimiento de la propia ciudad (Clark, Leavitt \& Williams, 2015), objetivo del juego hacia el que dirigían todos sus esfuerzos. Se promovía el uso compartido de recursos y contenidos del juego, potenciando con ello las relaciones sociales (Wohn, Lampe, Wash, Ellison \& Vitak, 2011).

El juego contaba con una interfaz gráfica muy intuitiva que apelaba a metáforas conocidas para hacer atractivo el escenario lúdico (Abdulhak, Cha \& Kang, 2011), y se ajustaba a los criterios de usabilidad y jugabilidad (Guegan, Moliner \& Buisine, 2015), facilitando la navegación e interacción. Mediante ventanas e intuitivos iconos se identificaban los datos de la ciudad y el nivel alcanzado por el jugador, visibilizando la energía, monedas, productos y disponibilidad de dinero, recursos acumulados para mejorar la ciudad, ampliar su población y terrenos, y el inventario de productos. Mostraba las tareas gratificadas, ofrecía nuevas misiones que proporcionaban energía adicional, permisos para expandirse, monedas y misiones de pago, retos para subir de nivel, etc.

\subsection{Mecánicas y dinámicas al servicio de la motivación}

Las reglas del juego establecían cómo construir la ciudad virtual, así como fórmulas de gratificación para garantizar la permanencia de los usuarios en el juego, y mecanismos para activar la interacción y colaboración entre ellos. La promoción de nivel se relacionaba con el aumento de población, lo que requería tiempo para construir nuevos

Perfil de jugador virtual asociado a la motivación personal para jugar en CityVille. M. Esther del Moral Pérez y Alba Patricia Guzmán Duque. 
edificios. Éstos tenían asignado un número de habitantes y conforme crecía la población se ampliaba la ciudad y sus servicios (cafeterías, hoteles, bancos, zona de ocio, etc.), con ello se subía de nivel, adquiriendo energía extra o dinero para ampliar el patrimonio virtual, reforzando su afán de superación para acometer nuevas tareas (Coelho, 2015).

Las fórmulas de gratificación contribuían a incrementar la motivación extrínseca de los usuarios y a fomentar su permanencia online al obtener energía, monedas, regalos de modo proporcional al tiempo que estuvieran jugando en su ciudad virtual, visitaran vecinos o participaran en el foro oficial. Así, se proponían retos al jugador para prolongar su estancia en el juego, sugiriendo nuevas misiones gratificadas, que permitían ampliar la ciudad añadiendo distritos comerciales, industriales, zonas nocturnas, etc. Ello proporcionaba reconocimiento social e incrementaba su prestigio al nombrarlo alcalde, gobernador, etc. (Lo, Lie \& Li, 2016).

La superación de nivel implicaba tareas más complejas para expandir la ciudad, aumentar los pobladores, construir centros comerciales, espacios lúdicos, etc. La interacción y la colaboración con otros se activaba mediante la gratificación de las visitas a sus ciudades, riego o recuperación de sembrados, entrega de obsequios y respuesta a solicitudes de vecinos, creación de franquicias y limpieza de barcos. La recaudación de dinero se obtenía a partir de la venta de productos, alquiler de edificios y casas, cosecha de los campos, visita a centros comerciales, etc., acumulando ganancias, nunca pérdidas. Igualmente, la comercialización de productos propios permitía el abastecimiento de negocios y la inversión en elementos de decoración, generando siempre ganancias (Coelho, Espinosa \& Medina, 2013).

La socialización entre jugadores se fomentaba al invitar a amigos de la red social y al promover las visitas a las ciudades de los jugadores colindantes para ofrecerles obsequios. Al tiempo, que se suscitaban prácticas basadas en la reciprocidad, al tener que corresponder con sus vecinos, devolviéndoles las visitas para obtener nuevas gratificaciones. Ello no sólo les permitía conocer sus puntuaciones, favorecer la competitividad y la auto-superación, sino que les ofrecía la oportunidad de aprender del diseño y las estrategias organizativas utilizadas por otros jugadores para planificar sus ciudades (Clark, Leavitt \& Williams, 2015). Los niveles superiores exigían una mayor participación y actividad de los jugadores.

Por otra parte, la publicación de peticiones (permisos y artículos para construir, energía, etc.) en el muro personal de otros jugadores, así como los ofrecimientos para realizar distintas tareas, emplearse en edificios comunitarios, etc., proporcionaban ganancias mutuas con el trueque de recursos o servicios. Igualmente sucedía al atender las solicitudes de ayuda de los vecinos a través de sus perfiles personales o mediante conversaciones entre jugadores vía chat en Facebook, Google+, etc. Este sistema de gratificación recíproca permitía adquirir regalos para la propia ciudad o donar artículos a los vecinos. La participación en el foro oficial del juego -en inglés, español y japonésfacilitaba el intercambio de experiencias y estrategias para expandir las ciudades, lo cual se premiaba con puntos (Lo, Lie \& Li, 2016).

Finalmente, hay que mencionar que si bien los usuarios podían jugar sin gastar dinero propio, se les invitaba tanto dentro del juego como vía Facebook a la compra de CityCash para obtener energía, productos, permisos o artículos para expandir la ciudad,

Perfil de jugador virtual asociado a la motivación personal para jugar en CityVille. M. Esther del Moral Pérez y Alba Patricia Guzmán Duque. 
incrementar su población y aumentar de nivel más rápidamente, esto suponía una microinversión económica fija asignada al juego para algunos (Kowert \& Oldmeadow, 2013).

\subsection{Jugar e interactuar en Cityville}

CityVille garantizaba la usabilidad y la creación de ciudades fantásticas (Jørgersen, 2012), cumplía con los requerimientos señalados por Bae, Koo y Mattila (2016), pues su escenario gráfico y la dinámica del juego contribuían a provocar el efecto inmersión o flow, implicando e integrando a los jugadores en ese mundo virtual, lo que les provoca una gran satisfacción al protagonizar las misiones propuestas (Reich y Vorderer, 2015). E incluso, suscitaba respuestas emocionales ante los estímulos presentados, al incorporar atributos que subrayaban su identidad personal, puntos alcanzados para establecer rankings, etc. (Tseng, Huang \& Teng, 2015).

Evidentemente, estos juegos en red logran cautivar y motivar a los usuarios, garantizando su permanencia y fidelización (Andreassen, et al., 2016), favoreciendo la interacción y socialización al generar experiencias de juego compartido (Corliss, 2011). CityVille incluía sorpresas para activar la motivación, la atención y la concentración del jugador, presentaba tareas atractivas y alentaba a la superación personal, incrementando progresivamente su dificultad para evitar el tedio y mantener el grado de complacencia de los jugadores con sus logros (Hughes, Griffin \& Worthington, 2017). Incorporaba sistemas para gratificar la permanencia en el juego y la culminación de las tareas, mostrando al jugador su propio avance, concediéndole libertad para decidir el tiempo dedicado al juego sin penalizarle, proponiéndole objetivos asequibles (Del Moral \& Guzmán, 2014), y retos novedosos para mantenerlos activos.

En general, la interacción social promovida por este tipo de juegos no sólo contribuye al incremento del capital social, -como sucedía en los juegos masivos multijugador online (MMOG), considerados sus precursores-, sino que pueden suscitar cambios en las conductas de los sujetos. Ante lo cual, Hussain, Williams y Griffiths (2015) alertan de que algunos usuarios adaptan su vida a estas fórmulas lúdicas, mostrando una preferencia desmedida por la interacción social en línea, lo que relacionan con una dependencia psicológica hacia el juego. En este sentido, existe una gran dicotomía, mientras unos investigadores como Hong, Hwang, Hsu, Tai y Kuo (2015), Lo, Lie y Li (2016), Vanwesenbeeck, Walrave y Ponnet (2016) aseguran que este tipo de juegos pueden provocar adicción, otros como Andreassen, et al. (2016), Chiang, et al. (2011), You, Kim y Lee (2017), O'Connor, Longman, White y Obst (2015), Lukavská, Hrabec y Chrz (2016) y Tseng, Huang y Teng (2015) los evalúan positivamente al entender que fomentan interacciones colaborativas enriquecedoras que se consolidan mediante la creación de comunidades de jugadores.

Clark, Leavitt y Williams (2015) señalan que estas plataformas lúdicas se usan masivamente por el interés y la flexibilidad que ofrecen para comunicarse, y las conceptúan como fórmulas de socialización atractivas al posibilitar la conectividad entre usuarios, e incrementar su confianza y aceptación social. Al tiempo que favorecen la adquisición de habilidades y destrezas cognitivas (Connolly, Boyle, MacArthur, Hainey \& Boyle, 2012; You, Kim \& Lee, 2017), propician múltiples aprendizajes derivados de tomas de decisiones y resolución de problemas planteados por el juego (Hou, 2012; Huang, Ali \& Liao, 2017); y suscitan cambios sustantivos en el aprendizaje social

Perfil de jugador virtual asociado a la motivación personal para jugar en CityVille. M. Esther del Moral Pérez y Alba Patricia Guzmán Duque. 
(Middaugh, 2016), generando lazos entre jugadores con intereses afines (Choi, Choi \& Song, 2012).

En CityVille se potenciaba la interacción para ganar, lo que contribuía a gestar una comunidad de jugadores orientada a la transferencia de estrategias para resolver los problemas derivados de las misiones de juego (Burroughs, 2014). Dando lugar a aprendizajes de diversa índole a partir de la colaboración mutua, con lo que alcanzaban los objetivos propuestos en el juego (Hussain, Williams \& Griffiths, 2015; O'Connor et al. 2015), ganaban reconocimiento social, al ser nombrado alcalde o gobernador e incrementaban su reputación social y popularidad (Kowert \& Oldmeadow, 2013).

\subsection{Motivaciones y perfiles de los jugadores virtuales}

Hay que destacar que uno de los alicientes que contribuyen a la fidelización de los usuarios a estos juegos virtuales es la oportunidad que se les ofrece para desempeñar y asumir determinados roles (Reich \& Vorderer, 2015). La persistencia en las tareas y su reconocimiento, la búsqueda de lo novedoso y la dependencia ligada a las recompensas otorgadas, etc., son elementos que activan la motivación de los jugadores. Éstos se sienten excitados ante la incertidumbre generada por el éxito o el fracaso de sus acciones, lo cual les obliga a prestar la máxima atención para tomar las decisiones más acertadas en cada jugada para ganar (Reich \& Vorderer, 2015).

Concretamente, Hussain, Williams y Griffiths (2015) remarcan seis factores que empujan a los usuarios a jugar: la novedad del juego, su rasgo social, el carácter del propio jugador, la competencia, el disfrute y el descubrimiento de nuevos escenarios. Para Huang, Huang, Chou y Teng (2017) y O'Connor et al. (2015), otro factor que lo afianza es la invitación a pertenecer a la comunidad oficial del juego, pues ello les proporciona un sentimiento de amparo al saberse respaldados por reglas explícitas. Su adscripción a estas comunidades les garantiza la resolución de problemas, el intercambio de experiencias entre jugadores, etc., favoreciendo su sensación de seguridad, lo que les alienta a seguir jugando online (Guegan, Moliner \& Buisine, 2015).

Sin duda, las notas hedonistas, funcionales y sociales que definen estos entornos lúdicos virtuales incrementan la vinculación de los usuarios al juego, sin olvidar la oportunidad que les ofrecen para desempeñar roles con los que se sienten a gusto (Huang, Ali \& Liao, 2017). Lo cual puede llegar a generar conductas adictivas en algunos sujetos (Kim, Kim, Shim, Im \& Shon, 2013), sin embargo Lukavská, Hrabec y Chrz (2016) señalan que esto no sucede si se les concede libertad para que dediquen el tiempo que deseen al juego sin ser penalizados, garantizándoles participar de manera autónoma.

En cualquier caso, las motivaciones personales de cada sujeto para permanecer en el juego son muy variadas, se relacionan con su carácter y ayudan a conformar un determinado perfil de jugador ligado, a pesar de todo no existe uniformidad al establecer categorías relativas específicamente a los juegos de construcción. Bae, Koo y Mattila (2016) indican que uno de los rasgos que definen los distintos perfiles de los jugadores virtuales está en consonancia con el tiempo que pasan jugando online, y ello se vincula con el margen de libertad y autonomía que se les concede para dedicarlo a esta actividad lúdica, así como su posibilidad de regresar al juego en cualquier momento.

Perfil de jugador virtual asociado a la motivación personal para jugar en CityVille. M. Esther del Moral Pérez y Alba Patricia Guzmán Duque.

Página 6 de 20 
Para Bourgonjon, Vandermeersche, De Wever, Soetaert y Valcke (2016) los jugadores se dividen en quienes solo quieren divertirse, y aquellos que dotan de un matiz prosocial al juego. Concretamente, los primeros invierten su tiempo para alcanzar reconocimiento social en el juego y disfrutar con ello, mientras los segundos además, buscan establecer vínculos y relaciones con otros usuarios, integrándose en las comunidades virtuales del juego al que pertenecen, primando el aprendizaje y el intercambio de estrategias. Así pues, la investigación que nos ocupa ha permitido acercarnos a los distintos perfiles de jugadores.

\section{Metodología}

\subsection{Contexto y objetivos de la investigación}

El presente estudio pretende constatar en qué medida las variables que contribuyen a definir la condición de jugador/a online, tales como sexo, tiempo dedicado al juego, nivel alcanzado, dinero invertido, etc., se relacionan con las motivaciones y los objetivos de los usuarios para permanecer el juego, así como con las razones para alegan para interactuar con otros jugadores en CityVille, y finalmente identificar los perfiles de jugador virtual más marcados. Para ello, se recabó información mediante una encuesta online dirigida a miembros de la comunidad de jugadores Fans-CityVille $(\mathrm{N}=110)$, que fue respondida por 105 sujetos. El procedimiento metodológico adoptó los criterios de Wohn et al. (2009) para obtener información sobre las relaciones generadas entre jugadores en red. La investigación se llevó a cabo en el curso 2014-15, antes de su cierre en marzo de 2015.

\subsection{Instrumento}

Se utilizó una cuenta de usuario en CityVille para invitar a jugadores activos a participar en una comunidad abierta en Facebook y posteriormente, a contestar una encuesta integrada por 12 ítems, 9 relativos a la dimensión perfil de jugador/a online $\mathrm{y}$, en concreto, de CityVille: sexo, edad, nacionalidad, cómo y cuándo lo conocieron, su frecuencia de juego, horas diarias dedicadas a jugar, nivel alcanzado y gasto mensual en el juego. Y otros 3 ítems, ligados a la dimensión motivación personal, sobre cuestiones más específicas que mostraban su grado de acuerdo -escala Likert (1-5)- con distintas afirmaciones sobre: a) sus motivaciones para jugar en CityVille; b) sus objetivos fijados con el juego; y, c) sus razones para interactuar con otros jugadores. La fiabilidad y la validez del instrumento se obtuvo mediante un alfa de Cronbach=0,93, adecuada para la investigación, tras su pilotaje con seis jugadores activos de CityVille, mayores de 35 años, para depurar su redacción.

\subsection{Procedimiento}

En primer lugar se presentan los datos descriptivos de la muestra de jugadores participantes en el estudio relativos a las dimensiones: perfil de jugador/a online en CityVille y motivación personal. Posteriormente, se aplican técnicas estadísticas multivariantes como ANOVA para analizar, concretamente, la incidencia de las variables sexo, tiempo dedicado al juego y nivel alcanzado en el juego, en las variables asociadas a la dimensión motivación personal (motivaciones, objetivos y razones para interactuar con otros en CityVille) (Figura 1), para constatar la existencia de diferencias significativas. E, igualmente se procede a partir de las correlaciones y de la regresión

Perfil de jugador virtual asociado a la motivación personal para jugar en CityVille. M. Esther del Moral 
múltiple. Para ello, se adoptaron los criterios metodológicos de Hair, Tatham, Anderson y Black (2010), y se utilizó el SPSS (v.18).

\begin{tabular}{|c|}
\hline $\begin{array}{c}\text { DIMENSIÓN } \\
\text { PERFIL DE JUGADOR DE CITYVILLE }\end{array}$ \\
\hline $\begin{array}{l}\text { V1. Sexo } \\
\text { V2. Edad } \\
\text { V3. Nacionalidad } \\
\text { V4. Actividad en juegos online } \\
\text { V5. Modo de acceso al juego }\end{array}$ \\
\hline $\begin{array}{l}\text { V6. Meses que lleva jugando } \\
\text { V7. Tiempo dedicado al juego } \\
\text { V8. Nivel alcanzado } \\
\text { v9. Gasto realizado en el juego }\end{array}$ \\
\hline
\end{tabular}

\section{MOTIVACIÓN PERSONAL PARA JUGAR EN CITYVILLE}

V10. Motivaciones para jugar en CityVille

V11. Objetivos fijados en el juego

V12. Razones para interaccionar con otros

Figura 1. Variables implicadas en el estudio.

\subsection{Muestra participante}

Respondieron voluntariamente 105 jugadores de la comunidad Fans-CityVille, una muestra integrada por un $62,9 \%$ hombres y $37,1 \%$ mujeres, procedentes de diferentes países: Colombia (33,3\%), Italia $(24,8 \%)$, México $(12,4 \%)$, España $(11,4 \%)$, Alemania (4.8\%), Canadá $(4,8 \%)$, Estados Unidos $(3,8 \%)$ y el $4,7 \%$ restante a otros países (Arabia, Angola, Brasil, Paraguay y Austria). El 1,0\% era menor de 18 años, el 7,6\% entre 18-25 años, el 22,9\% entre 26-35, el 44,8\% entre 36-45 años, un 20,0\% entre 46$55 \mathrm{y}$ un $3,8 \%$ mayor de 55 .

En relación a su actividad en juegos online, los encuestados se consideran jugadores activos, si bien un 16,2\% sólo está registrado en este juego, un 51,4\% está entre 2-4 juegos más, un $23,8 \%$ entre $5-7$; un $5,7 \%$ entre $8-10$, y un $2,9 \%$ en más 10 juegos. Y mientras, el 94,3\% se declara jugador habitual, únicamente un 5,7\% manifiesta jugar una vez a la semana. Por otro lado, el 93,3\% indica que accedió a CityVille invitado por conocidos o amigos de Facebook, un marginal 2,9\% entró por curiosidad, y otro 2,9\% inducido por la publicidad. Un 1,0\% refiere la invitación a familiares.

\section{Resultados}

\subsection{Dimensión perfil de jugador de CityVille}

La descripción de la muestra, atendiendo a las variables que contribuyen a explicar su condición de jugadores online en CityVille, queda del siguiente modo:

a) Tiempo:

- Meses que lleva jugando. El 44,8\% lleva jugando entre 13-18 meses, seguidos del 29,5\% que lleva entre 6-12 meses, un 18,1\% menos de 6 meses, y un 7,6\% entre 19-24 meses.

Perfil de jugador virtual asociado a la motivación personal para jugar en CityVille. M. Esther del Moral 
- Horas dedicadas al juego. Mientras un 18,1\% juega menos de una hora diaria, el 30,5\% dedica entre 1 y 2 horas al día, otro 30,5\% juega entre 3 y 5 horas; y un nada desdeñable 16,2\% manifiesta dedicar más de 5 horas diarias. Solo un 2,9\% declara jugar esporádicamente 1 vez por semana, junto al 1,9\% que lo hace 2 veces por semana.

\section{b) Nivel alcanzado en el juego.}

- El 43,8\% supera el nivel 100, el 29,5\% se sitúa entre el 51-100; un 11,4\% posee niveles entre 11-20; un 9,5\%, localizado entre $21-50$ y otro $9,5 \%$ supera el 200. Sólo el 2,9\% posee niveles entre 6-10; los de niveles inferiores (1-5) sólo representan el 1,9\%. Hay que tener en cuenta que no hay tope de nivel, se juega y se promociona sin fin.

c) Gasto.

- El 52,4\% afirma no invertir dinero en el juego, mientras el 47,6\% restante confiesa tener un gasto fijo mensual. Concretamente, un $19 \%$ invierte entre 2,31 y $5,75 \$$, seguido por un $13,3 \%$ que afirma gastar entre 5,76 y 11,50 \$; un 8,6\% invierte lo mínimo establecido en el juego $(2,30 \$) ;$ y un $6,7 \%$ cifra su gasto entre 11,51 y $23 \$$.

\subsection{Dimensión motivación personal}

Las motivaciones personales de los sujetos para permanecer horas conectados al juego son muy diversas y, en ocasiones, pueden implicar ciertas conductas adictivas. Así, partiendo de la formulación de hipótesis similares a las de Snodgrass et al. (2012), se quiso analizar cómo las distintas motivaciones modelaban el perfil de los jugadores. Para ello, se consultó a los jugadores de CityVille $(C V)$ al respecto, constatando sus distintas respuestas: necesidad de divertirse o evadirse, sentirse feliz, satisfechos con los logros alcanzados en el juego, e incluso, con su convicción de sentirse realizados/as con ello.

\subsubsection{Diversión, felicidad y realización personal}

Así pues, en la gráfica 1 se observa la distribución de los jugadores en función de su grado de acuerdo (1 nada-5 muy de acuerdo) con las motivaciones que les empujan a jugar en $C V$, si bien para facilitar la presentación, los valores se agrupan en: 1-2 (nada y poco de acuerdo), 3 de acuerdo, 4-5 (bastante y muy de acuerdo). Concretamente, se constata que el $96,2 \%$ dice estar bastante o muy de acuerdo con que juega para divertirse, un 89,5\% afirma sentirse feliz al jugar, y el 88,6\% está bastante de acuerdo al afirmar sentirse satisfecho/a al hacerlo. Si bien al analizar su grado de acuerdo sobre si ello les hace sentirse realizados, hay más dispersión en sus respuestas, un 54,3\% manifiesta estar bastante o muy de acuerdo y un 33,3\% sólo de acuerdo. 


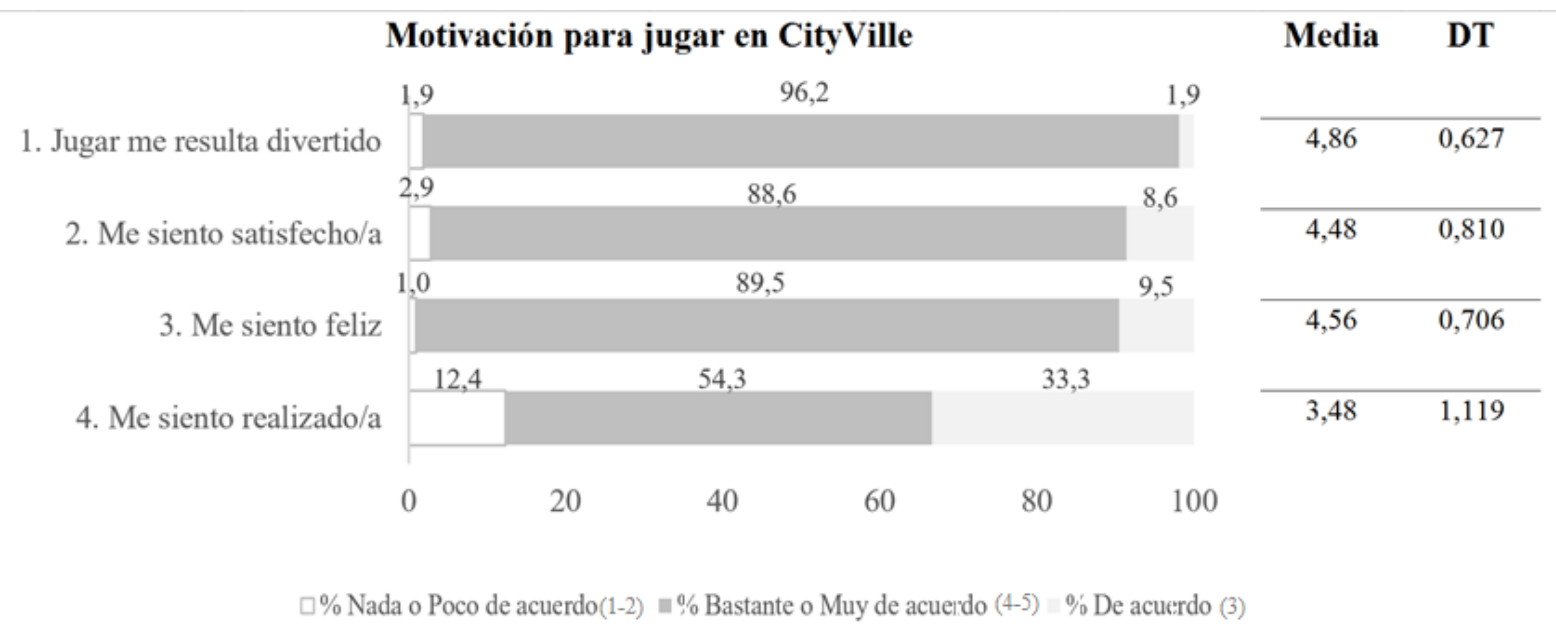

Gráfica 1. Motivaciones para jugar en CV. Elaboración propia.

Las correlaciones muestran que las horas diarias dedicadas a jugar en CV se relacionan positivamente con la mayoría de sus motivaciones $(\mathrm{W}=0,181)$, así como con el nivel alcanzado en el juego. La regresión múltiple permitió identificar las dos motivaciones principales alegadas por los jugadores para permanecer en $\mathrm{CV}$ que contribuyen a explicar un mayor gasto en el mismo: sentirse felices cuando juegan y sentirse realizados $(\mathrm{R} 2=0,240, \mathrm{p}<0,000)$. Sin embargo, tras el ANOVA no se detectaron diferencias significativas en las motivaciones reseñadas por los jugadores respecto a la variable sexo. Tampoco el sexo influía en su actividad como jugador, ni en los niveles alcanzados en el juego.

\subsubsection{Perfiles ligados a las prioridades establecidas en el juego}

Al preguntar a los usuarios por los objetivos establecidos para jugar, se obtuvieron respuestas que permitieron identificar su perfil, para algunos más utilitarista y adictivo, relacionados con su afán por avanzar más rápido en los niveles, obtener más XP para subir de nivel, obtener energía para jugar más, construir la ciudad de sus sueños o sentirse propietarios poderosos en el mundo virtual. Para otros, un perfil ligado al aprendizaje y la colaboración, al ponderar las oportunidades ofrecidas por el juego para aprender colaborando: obtener elementos para construir, colaborar con obsequios y regalos y ayudar a los vecinos a expandir su ciudad. Y un perfil, más prosocial, propio de los que resaltan la posibilidad de socialización que implica el juego, considerando compartir estrategias y/o solicitar ayuda a sus vecinos (Gráfica 2). 


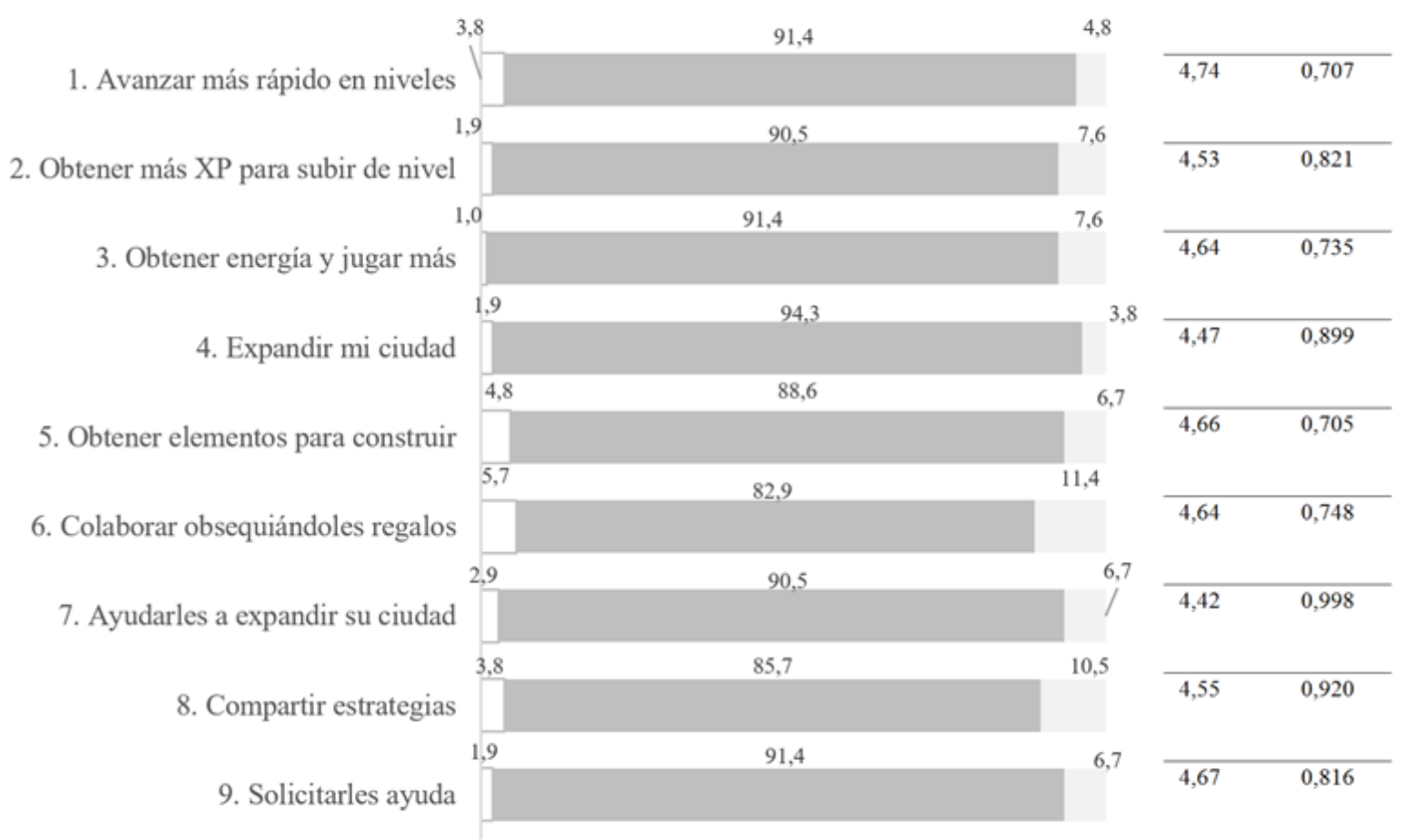

$\square \%$ Nada o Poco de acuerdo (1-2) $=\%$ Bastante o Muy de acuerdo (4-5) \% De acuerdo (3)

Gráfica 2. Objetivos priorizados para jugar en CV. Elaboración propia.

La mayoría $(98,1 \%)$ afirma que juega por diversión, el matiz adictivo viene marcado por otras afirmaciones pues, a su vez el 93,3\% declara hacerlo para obtener energía, interactuar y tener algo propio aunque sea virtual. Otro 91,4\%, lo vincula a la posibilidad de construir la ciudad de sus sueños, compartir afición con otros, adquirir nuevas estrategias y contactar con otros. Y un $89,5 \%$, lo relaciona con la oportunidad ofrecida por el juego para conocer gente.

Con todo, las correlaciones muestran que las horas dedicadas al juego se relacionan positivamente con la mayoría de los objetivos que los jugadores priman, lógicamente un mayor tiempo de juego garantiza el logro de sus objetivos en mayor medida. Además, también se relaciona con el nivel alcanzado en el juego ( $\mathrm{W}>0,213, \mathrm{p}<0,015$ ). Asimismo, la regresión múltiple muestra que si bien todos los objetivos se correlacionan positiva y significativamente con la variable gasto en el juego $(\mathrm{R} 2=0,135, \mathrm{p}<0,000)$, se detecta que el objetivo principal de los jugadores para gastar dinero en $\mathrm{CV}$ es promocionar de nivel más rápido $(\mathrm{R} 2=0,107, \mathrm{p}<0,001)$. Así, se constató que los usuarios que gastaban más dinero, jugaban más horas diarias $\mathrm{y}$, en consecuencia, alcanzaban mayores niveles $(\mathrm{R} 2=0,352, \mathrm{p}<0,000)$. Por contra, quienes no invertían dinero no jugaban tanto tiempo. El promedio de los jugadores dedicaba entre 1-2 horas diarias al juego (32,7\%). Y el 36,4\% alcanzó niveles entre el 51-100, el 21,8\% entre 1120 , y el $18,2 \%$ entre $21-50$.

Al realizar la regresión se detecta que el dinero gastado en el juego se relaciona con la variable sexo $(p<0,000)$, siendo ellas quienes invierten más dinero $(\mathrm{p}<0,027)$. Con el 
ANOVA se evidenció la existencia de diferencias significativas entre los objetivos fijados por las mujeres y los de los hombres con el juego, mientras ellas prefieren compartir estrategias $(\mathrm{p}<0,009)$, ellos priorizan la obtención de energía para subir de nivel $(\mathrm{p}<0,068)$.

\subsubsection{Ganar versus compartir}

Indudablemente, este tipo de juegos en red añade un matiz distintivo a los videojuegos convencionales al arbitrar fórmulas y herramientas diversas que facilitan la comunicación entre los usuarios. Además, en CityVille se fomentaban las interacciones y se gratificaban con la adquisición de recursos para seguir jugando y alcanzar mayores cotas de poder. Sin embargo, el factor individual es el que marca las relaciones entre los sujetos, de ahí que a los encuestados se les pidiera que expresaran las razones que les movían a interactuar con sus compañeros o vecinos del juego. Las cuales se han podido alinear, permitiendo vislumbrar los perfiles mencionados: a) utilitarista-adictivo, ligado a la obtención de energía para jugar más, tener algo propio aunque sea virtual, construir la ciudad de mis sueños y divertirse; b) de aprendizaje lúdico-colaborativo: aprender nuevas estrategias, mantener contacto con amigos, conocer a nuevas personas y compartir estrategias; y c) prosocial, buscando interactuar y compartir afición con otros (Gráfica 3).

\section{Razones para interactuar en CityVille}

\section{Media DT}

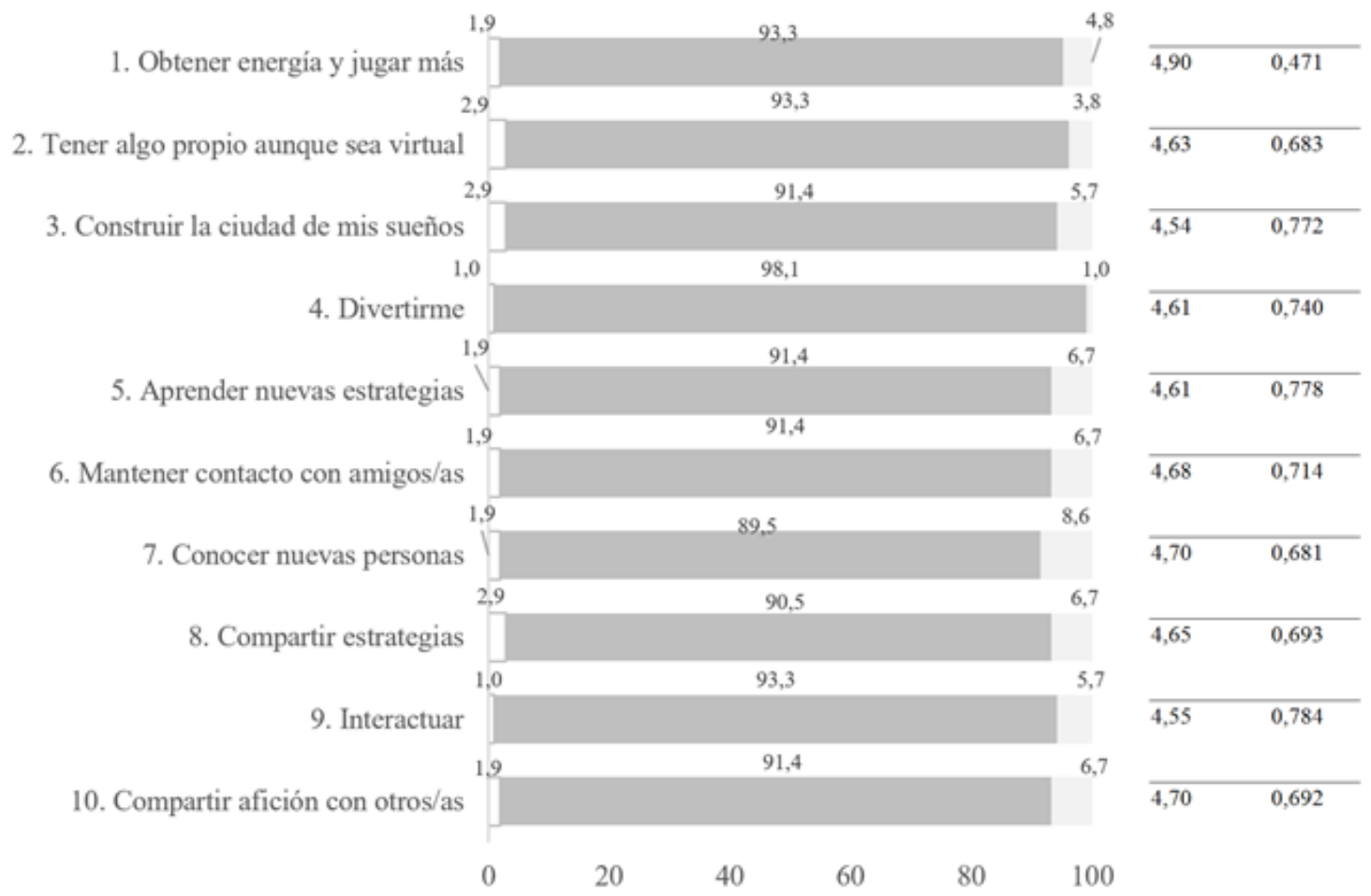

$\square \%$ Nada o Poco de acuerdo(1-2) $=\%$ Bastante o Muy de acuesdo (4-5) \% De acuerdo (3)

Gráfica 3. Razones para interactuar con otros en CV. Elaboración propia.

Los encuestados afirman estar bastante o muy de acuerdo $(94,3 \%)$ al señalar que una de las prioridades para interactuar con sus vecinos es expandir su propia ciudad, -actitud 
recompensada por el juego- y es la razón que alega el 91,4\%, puesto que eso les aporta un avance más rápido, obtención de energía y más juego, seguido del 90,5\% que lo justifica dado que les ayuda a expandir su ciudad y a obtener más energía para promocionar de nivel. Planteamientos netamente competitivos que son reforzados por el juego e, incluso, algo utilitaristas, dado que se generan relaciones entre jugadores de interdependencia condicionadas por intereses individuales. Lo que se refleja en sus apreciaciones, pues el 91,4\% interactúa con otros jugadores para solicitarles ayuda $(91,4 \%)$ o elementos para construir $(88,6 \%)$, mientras un $82,9 \%$ lo hace para ayudarles a expandir su ciudad u obsequiarles con regalos.

Indudablemente, este juego fomentaba la adquisición de múltiples aprendizajes a partir de su experiencia de juego, logrando activar numerosas estrategias que les permitía optimizar el proceso de construcción y expansión de su propia ciudad. Así lo considera el $85,7 \%$ al afirmar que interactúa con sus vecinos para compartir estrategias.

El análisis correlacional detecta que las horas diarias dedicadas al juego se relacionan positivamente con las razones alegadas por los jugadores para interactuar con otros, con su nivel alcanzado (W > 0,177). Lógicamente, también lo hacen con su condición de jugadores habituales, con el tiempo que llevan en el juego y con el dinero que dicen gastar mensualmente ( $\mathrm{W}=0,226)$. Por lo tanto, a mayor inversión mayor es el nivel alcanzado en el juego ( $\mathrm{W}>0,545 ; \mathrm{p}<0,000)$. Igualmente, con la regresión múltiple se detectó que la variable sexo se relaciona con el nivel de juego $(\mathrm{R} 2=0,335, \mathrm{p}<0,013)$, siendo los hombres quienes alcanzan niveles superiores.

Para constatar la existencia de diferencias significativas entre las razones alegadas por hombres y mujeres para interactuar con otros jugadores, se efectuó un ANOVA, donde se observó que ellas priman la interacción con sus vecinos para ayudarles a expandir su ciudad y para compartir estrategias ( $\mathrm{p}<0,040)$, mientras que los hombres lo hacen en tanto les garantiza construir la ciudad de sus sueños, tener algo propio aunque sea virtual y adquirir nuevas estrategias ( $\mathrm{p}<0,000)$.

\section{Discusión}

La comunidad de jugadores encuestada para este estudio estaba integrada tanto por hombres como por mujeres, si bien había más hombres (62,9\%). Se observa una constelación de satélites alrededor del jugador italiano que posee el mayor nivel y, lógicamente, entre sus vecinos se localizan jugadores de su mismo país, amigos de sus amigos y, de forma semejante, se encuentra un grupo de usuarios de Colombia, rodeado de su círculo de amistades.

La media de edad de los miembros de la comunidad Fans-CityVille era de 40 años $(44,8 \%)$. Algo más de la mitad (51,4\%) indicaba jugar al menos en 3 juegos online más, un 44,8\% llevaba jugando en CityVille casi 15 meses, tiempo considerablemente largo que refleja su poder de fidelización. Además, mientras un 30,5\% indicó que jugaba diariamente entre 1-2 horas, un 18,1\% dedicaba entre 3-5 horas al juego. En consonancia con ello, un 43,8\% mencionó haber alcanzado niveles entre el 90-100 en el juego. Con porcentajes similares, el 47,6\% de los usuarios aseguró invertir un promedio de 8 dólares al mes, mientras el 19,0\% invertía el mínimo contemplado en el juego, es decir, entre 2,30 y 5,75 dólares. 
El análisis correlacional permitió comprobar cómo las motivaciones de los usuarios para permanecer en CityVille -divertirse, sentirse feliz y realizados/as con el juego- influían de manera positiva en el logro de los niveles más altos, e indudablemente contribuían a garantizar un avance más rápido. Igualmente, se constató que los objetivos que se fijaban los jugadores -divertirse, tener algo propio aunque sea virtual, interactuar con otros, expandir su ciudad...- se relacionaban con su afán por lograr niveles superiores. Asimismo, se obtuvo una correlación positiva entre el nivel alcanzado en el juego y las razones alegadas para interactuar con otros jugadores.

Respecto al gasto, como era de esperar, se comprobó que los usuarios que más dinero invertían eran los que dedicaban más horas al día a jugar, y por consiguiente, alcanzan los niveles más altos, detectándose curiosamente que eran las mujeres las que decían gastar más ( $\mathrm{p}<0,027)$. A tenor de los datos, se puede concluir que los que más jugaban más gastaban $(\mathrm{R} 2=0,352)$, dado que la inversión de dinero garantizaba una promoción más rápida. Lo que contribuía a afianzar su empeño por sentirse satisfechos, y a alcanzar el reconocimiento social que esto podía suponer entre su entorno de socialización virtual, su grupo de amistades en las redes sociales, vecinos en el juego, etc.

De las respuestas de los jugadores se infiere que además de divertirse y ganar -vertiente más adictiva y competitiva-, también deseaban activar su imaginación y creatividad al expandir sus ciudades, aprender de otros compartiendo estrategias $(85,7 \%)$, y conocer a personas con intereses afines. La mayoría buscaba interaccionar con sus vecinos en el juego para colaborar y solicitaban su ayuda con distintas finalidades. Unos pocos -desde una perspectiva más utilitarista- lo hacían para obtener energía o puntos adicionales para subir de nivel más rápido y construir una ciudad de grandes dimensiones. A otros les gustaba compartir experiencias y trucos, mientras algunos se sentían cómodos integrándose en la comunidad de usuarios dado que les permitía socializarse.

A ese respecto, se detectaron diferencias significativas tanto en los objetivos que se fijaban hombres y mujeres, como en las razones alegadas para interactuar con otros jugadores. Las mujeres preferían compartir estrategias, mientras los hombres buscaban obtener más energía para promocionar de nivel, razón por la que alcanzaban niveles más altos que sus compañeras. Finalmente, si bien las mujeres primaban la interacción con sus vecinos para ayudarles a expandir su ciudad y para compartir estrategias, los hombres lo hacían en la medida que ello les garantizaba la construcción de la ciudad de sus sueños, tener algo propio aunque sea virtual y adquirir nuevas estrategias.

\section{Conclusiones}

Como se ha podido observar, las mecánicas y dinámicas de CityVille daban lugar a prácticas de juego que confrontaban el deseo de ganar de los usuarios, de tener algo como propio y de subir de nivel, con el hecho de compartir experiencias y estrategias con otros jugadores. A pesar de todo, este juego lograba conciliar ambas posiciones de un modo acertado, al relacionar la promoción de nivel con la interacción y la ayuda mutua entre los usuarios. En concreto, el componente prosocial del juego quedaba plasmado en las reglas específicas que establecía al gratificar las interacciones entre los jugadores, asignando puntos por cada visita efectuada a las ciudades de los vecinos, al

Perfil de jugador virtual asociado a la motivación personal para jugar en CityVille. M. Esther del Moral Pérez y Alba Patricia Guzmán Duque. 
dar respuesta a sus solicitudes de ayuda, etc., favoreciendo el crecimiento de las ciudades de ambos y el avance de nivel, reforzando positivamente su motivación personal.

Tras el análisis, se constata que de forma más o menos directa, las actividades lúdicas propuestas por este tipo de juegos orientados a la construcción, en este caso de ciudades, favorecen la definición de distintos perfiles de jugador, en concreto, aquí se han identificado tres: utilitarista-adictivo, de aprendizaje-lúdico colaborativo o prosocial. Aunque existen variables individuales, propias de cada sujeto, que van a condicionarlos en mayor medida, se observa que estos perfiles se asocian tanto a las motivaciones que les impulsa a jugar, como a los objetivos personales que se proponen, y al valor que confieren a las interacciones virtuales en el escenario lúdico.

Así pues, los sujetos que responden a un perfil utilitarista-adictivo subrayan la necesidad de sentirse propietarios de una ciudad virtual, para lo cual orientan todos sus esfuerzos a la obtención de energía para avanzar más rápido en los niveles y construir la ciudad de sus sueños, por lo que la interacción y comunicación con otros jugadores la consideran un medio más para alcanzar sus objetivos. Todo ello puede contribuir a que esta actividad se convierta en adictiva para algunos, dado el elevado número de horas diarias que dedican al juego y el presupuesto que asignan al mismo, en pro de alcanzar mayores cotas de poder y reconocimiento social. Y, si bien se trata de una minoría dentro de la comunidad analizada, no deja de ser preocupante que un 30,5\% jugara entre 3 y 5 horas diarias e invirtiera 23 dólares mensuales.

Por su parte, aquellos que se ajustan a perfiles que priman el aprendizaje a partir de prácticas de entretenimiento colaborativo, como era el caso de la construcción de ciudades virtuales, se ha podido constatar que buscaban la relación con otros jugadores a través de las visitas a sus ciudades para compartir experiencias y aprender de sus estrategias. Lo cual permitía que se vieran beneficiadas ambas partes, no sólo por las puntuaciones añadidas que lograban con ello, sino por la oportunidad que les brindaba el juego tanto para contemplar los diseños creativos de otros como para desarrollar su creatividad. Se trata de un perfil que pondera la colaboración y la ayuda mutua, y curiosamente se corresponde en mayor medida con los jugadores de género femenino, a tenor de las motivaciones que les impulsan a jugar, sus objetivos y razones alegadas para comunicarse con otros.

De forma semejante, aunque con ciertos matices, el perfil de jugador prosocial se enfoca a aprovechar los recursos, las herramientas (foros, comunidad de jugadores, etc.) y las dinámicas del propio juego -tales como las interacciones que promovía-, etc., para activar la socialización con el resto de jugadores. Ya sea a partir del intercambio de estrategias, mediante la interacción directa durante el juego o generando una comunicación asíncrona para solicitar u ofrecer ayuda (trucos para resolver problemas derivados del crecimiento de las propias ciudades o estrategias para avanzar más rápido). Son los usuarios que generan actividad social, dinamizan la comunidad, e incluso, en el periodo crítico de cierre del juego (marzo 2015) tuvieron un papel relevante manifestando su frontal oposición. Así, tras analizar esta comunidad se comprobó que las mujeres se ajustaban más a este perfil que los hombres. 
Finalmente, se puede afirmar que las diferencias significativas en función del género encontradas entre los usuarios subrayan sus distintos perfiles en tanto jugadores. Las mujeres se ajustaban más a perfiles que primaban un entretenimiento colaborativo y prosocial, enfocado a la interacción con otros usuarios para compartir estrategias. Mientras, los hombres destacaban por perfiles más utilitaristas -e incluso, adictivosguiados por motivaciones de carácter competitivo, enfocados a ganar, por lo que orientaban todos sus esfuerzos al crecimiento de sus ciudades virtuales.

Presentación del artículo: 13 de febrero de 2017 Fecha de aprobación: 15 de marzo de 2017 Fecha de publicación: 30 de junio de 2017

Del Moral Pérez, M.E., y Guzmán Duque, A.P. (2017). Perfil de jugador virtual asociado a la motivación personal para jugar en CityVille. RED. Revista de Educación a Distancia, 54. Consultado el (dd/mm/aaaa) en http://www.um.es/ead/red/54/

\section{FINANCIACIÓN}

El presente trabajo se inserta en el proyecto de investigación denominado E3M "European Indicator and Ranking Methodology for University Third Mission", en el Centro de Gestión de la Calidad y del Cambio de la Universitat Politècnica de València (UPV).

FUNDING

This paper is included within the research project titled "European Indicator and Ranking Methodology for University Third Mission", recognized by Centro de Gestión de la Calidad y del Cambio de la Universitat Politècnica de València (UPV).

\section{Referencias bibliográficas}

Abdulhak, S. A., Cha, K. H., \& Kang, D. K. (2011, November). Evaluation of online social network games usability using verbal protocol analysis. In Computer Sciences and Convergence Information Technology (ICCIT), 2011 6th International Conference on (pp. 7-11). IEEE.

Andreassen, C. S., Billieux, J., Griffiths, M. D., Kuss, D. J., Demetrovics, Z., Mazzoni, E., \& Pallesen, S. (2016). The relationship between addictive use of social media and video games and symptoms of psychiatric disorders: A large-scale crosssectional study. Psychology of Addictive Behaviors, 30(2), 252-262. http://dx.doi.org/10.1037/adb0000160

Perfil de jugador virtual asociado a la motivación personal para jugar en CityVille. M. Esther del Moral 
Bae, J., Koo, D. M., \& Mattila, P. (2016). Affective motives to play online games. Journal of Global Scholars of Marketing Science, 26(2), 174-184.

Berle, D., Starcevic, V., Porter, G., \& Fenech, P. (2015). Are some video games associated with more life interference and psychopathology than others? Comparing massively multiplayer online role-playing games with other forms of video game. Australian Journal of Psychology, 67(2), 105-114.

Bourgonjon, J., Vandermeersche, G., De Wever, B., Soetaert, R., \& Valcke, M. (2016). Players' perspectives on the positive impact of video games: A qualitative content analysis of online forum discussions. New Media \& Society, 18(8), 1732-1749.

Burroughs, B. (2014). Facebook and FarmVille A Digital Ritual Analysis of Social Gaming. Games and Culture, 9(3), 151-166. Doi: 10.1177/1555412014535663

Camilleri, V., Busuttil, L., \& Montebello, M. (2011). Social Interactive Learning in Multiplayer Games. In: Ma, M., Oikonomou, A., Jain, L.C. (eds.). Serious Games and Edutainment Applications. London: Springer-Verlag.

Chiang, Y. T., Lin, S. S., Cheng, C. Y., \& Liu, E. Z. F. (2011). Exploring Online Game Players' Flow Experiences and Positive Affect. Turkish Online Journal of Educational Technology-TOJET, 10(1), 106-114.

Choi, H., Choi, E., \& Song, S. (2012). The structure of affection descriptors for social network game: case study of CityVille on Facebook. Multimedia Tools and Applications, 68(2), 375-389. Doi: 10.1007/s11042-012-1310-y

Clark, J., Leavitt, A., \& Williams, D. (2015). Online Games, Community Aspects of. The International Encyclopedia of Digital Communication and Society, 1-7.

Coelho, P., M. F., Espinosa, R., S. C., \& Medina, I. G. (2013). O game CityVille: uma nova tendência da Web/The CityVille Game: A New Trend on the Web. Revista EducaOnline, 7(2), 18-33.

Coelho, P., M. F. (2015). Um Olhar Semiótico Sobre Game CityVille do Facebook. Revista Hipertexto, 5(1), 19-52.

Cole, H., \& Griffiths, M.D. (2007). Social interactions in massively multiplayer online role-playing gamers. Cyberpsychology \& Behavior, 10(4), 575-583. Doi: 10.1089/cpb.2007.9988

Connolly, T.M., Boyle, E.A, MacArthur, E., Hainey, T., \& Boyle, J. (2012). A systematic literature review of empirical evidence on computer games and serious games. Computers \& Education, 59(2), 661-686. Doi: 10.1016/j.compedu.2012.03.004 
Corliss, J. (2011). Introduction: The Social Science Study of Video Games. Games \& Culture, 6(1), 3-16. Doi: 10.1177/1555412010377323

Correa, A. M. (2016). Los juegos sociales como conversaciones mediadas en el mercadeo relacional: Mentez.com, el caso latinoamericano. Revista Conciencia Empresarial, 2(5), 101-119.

De Andrade, S. (June, 2012). Buy and Share! Social Network Games and Ludic Shopping. DiGRA Nordic 2012 Conference: Local and Global-Games in Culture and Society, vol. 10. Tampere, Finland.

Del Moral, M.E y Guzmán, A.P. (2014). CityVille: collaborative game play, communication and skill development in social networks. Journal of New Approaches in Educational Research, 3(1), 11-19. DOI:10.7821/naer.3.1.11-19

Feinleib, D. (2011). Why Startups Fail: And How Yours Can Succeed. London: Apress.

Granic, I., Lobel, A., \& Engels, R.C. (2013). The Benefits of Playing Video Games. American Psychological Association, 69(1), 66-78. Doi: 10.1037/a0034857

Guenaga, M., Eguíluz, A., Jerez, A. R., \& Torientes, E. Q. (2015). Un juego Serio para Desarrollar y Evaluar la Competencia de Trabajo en Equipo. IE Comunicaciones: Revista Iberoamericana de Informática Educativa, 21, 3-11.

Guegan, J., Moliner, P., \& Buisine, S. (2015). Why are online games so self-involving: A social identity analysis of massively multiplayer online role-playing games. European Journal of Social Psychology, 45(3), 349-355.

Hair, J.F., Tatham, R. L., Anderson, R. E., \& Black, W. (2010). Análisis multivariante. Madrid: Prentice Hall.

Hou, H.T. (2012). Exploring the behavioral patterns of learners in an educational massively multiple online role-playing game (MMORPG). Computers \& Education, 58(4), 1225-1233. Doi: 10.1016/j.compedu.2011.11.015

Huang, H. C., Huang, L. S., Chou, Y. J., \& Teng, C. I. (2017). Influence of Temperament and Character on Online Gamer Loyalty: Perspectives from Personality and Flow Theories. Computers in Human Behavior, 70, 398-406. doi.org/10.1016/j.chb.2017.01.009.

Huang, M., Ali, R., \& Liao, J. (2017). The effect of user experience in online games on word of mouth: A pleasure-arousal-dominance (PAD) model perspective. Computers in Human Behavior, 75, 329-338.

Hughes, C. M., Griffin, B. J., \& Worthington, E. L. (2017). A measure of social behavior in team-based, multiplayer online games: The Sociality in Multiplayer Online Games (SMOG) scale. Computers in Human Behavior, 69, 386-395.

Perfil de jugador virtual asociado a la motivación personal para jugar en CityVille. M. Esther del Moral Pérez y Alba Patricia Guzmán Duque. 
Hussain, Z., Williams, G. A., \& Griffiths, M. D. (2015). An exploratory study of the association between online gaming addiction and enjoyment motivations for playing massively multiplayer online role-playing games. Computers in Human Behavior, 50, 221-230.

Jørgersen, K. (2012). Between the Game System and the Fictional World: A Study of Computer Game Interfaces. Games and Culture, 7(2), 142-163. Doi: $10.1177 / 1555412012440315$

Kim, P.W., Kim, S.Y., Shim, M., Im, C.H., \& Shon, Y. M. (2013). The influence of an educational course on language expression and treatment of gaming addiction for massive multiplayer online role-playing game (MMORPG) players. Computers \& Education, 63, 208-217. Doi: 10.1016/j.compedu.2012.12.008

Kowert, R., \& Oldmeadow, J. A. (2013). (A) Social reputation: Exploring the relationship between online video game involvement and social competence. Computers in Human Behavior, 29(4), 1872-1878.

Lo, S. K., Lie, T., \& Li, C. L. (2016). The relationship between online game playing motivation and selection of online game characters-the case of Taiwan. Behaviour \& Information Technology, 35(1), 57-67.

Lukavská, K., Hrabec, O., \& Chrz, V. (2016). The Role of Habits in Massive Multiplayer Online Role-Playing Game Usage: Predicting Excessive and Problematic Gaming Through Players' Sensitivity to Situational Cues. Cyberpsychology, Behavior, and Social Networking, 19(4), 277-282.

Hong, J. C., Hwang, M. Y., Hsu, C. H., Tai, K. H., \& Kuo, Y. C. (2015). Belief in dangerous virtual communities as a predictor of continuance intention mediated by general and online social anxiety: the Facebook perspective. Computers in Human Behavior, 48, 663-670.

Middaugh, E. (2016). The Social and Emotional Components of Gaming. A Response to "The Challenge of Gaming for Democratic Education". Democracy and Education, 24(2), 1-12.

Nummenmaa, T., Alha, K., \& Kultima, A. (2011). Towards Game Evolution Planning Through Simulation. En: Kultima, A.; Peltoniemi, M. (eds). Games and Innovation Research Seminar 2011 Working Papers. TRIM Research Reports 7. Tampere, Finland: School of Information Sciences, University of Tampere.

O'Connor, E. L., Longman, H., White, K. M., \& Obst, P. L. (2015). Sense of community, social identity and social support among players of Massively multiplayer online games (MMOGs): A qualitative analysis. Journal of Community \& Applied Social Psychology, 25(6), 459-473.

Reich, S., \& Vorderer, P. (2015). Online Games, Player Experiences in. The International Encyclopedia of Digital Communication and Society, 1-12. Recuperado 
https://www.researchgate.net/profile/Sabine_Reich/publication/272828623_Onli ne_Games_Player_Experiences_in/links/54f323740cf299c8d9e4f85e.pdf

Snodgrass, J.G., Dengah, H.F., Lacy, M.G., Fagan, J., Most, D., ... \& Wintersteen, B. (2012). Restorative magical adventure or warcrack? Motivated MMO play and the pleasures and perils of online experience. Games and Culture, 7(1), 3-28. Doi: $10.1177 / 1555412012440312$

Tseng, F. C., Huang, H. C., \& Teng, C. I. (2015). How do online game communities retain gamers? Social presence and social capital perspectives. Journal of Computer-Mediated Communication, 20(6), 601-614.

Vanwesenbeeck, I., Walrave, M., \& Ponnet, K. (2016). Young adolescents and advertising on social network games: a structural equation model of perceived parental media mediation, advertising literacy, and behavioral intention. Journal of Advertising, 45(2), 183-197.

Wohn, D. Y., Lampe, C., Wash, R., Ellison, N., \& Vitak, J. (2011). The "S" in Social Network Games: Initiating, Maintaining, and Enhancing Relationships. The 44th Hawaii International Conference on System Sciences, Hawaii, USA.

You, S., Kim, E., \& Lee, D. (2017). Virtually real: exploring avatar identification in game addiction among massively multiplayer online role-playing games (MMORPG) players. Games and Culture, 12(1), 56-71.

Zynga. (2013). Información corporativa de Zynga. Recuperado de https://www.zynga.com/ 